\title{
Singularity Processing Method of Microstrip Line Edge Based on LOD-FDTD
}

\author{
Lei Li, ${ }^{1}$ Xin Zhang, ${ }^{1,2}$ and Yingsong $\mathrm{Li}^{1}$ \\ ${ }^{1}$ College of Information and Communications Engineering, Harbin Engineering University, Harbin 150001, China \\ ${ }^{2}$ School of Information Engineering, Wuyi University, Jiangmen 529020, China
}

Correspondence should be addressed to Yingsong Li; liyingsong@ieee.org

Received 16 October 2014; Revised 2 December 2014; Accepted 8 December 2014; Published 25 December 2014

Academic Editor: Ananda S. Mohan

Copyright (C) 2014 Lei Li et al. This is an open access article distributed under the Creative Commons Attribution License, which permits unrestricted use, distribution, and reproduction in any medium, provided the original work is properly cited.

In order to improve the performance of the accuracy and efficiency for analyzing the microstrip structure, a singularity processing method is proposed theoretically and experimentally based on the fundamental locally one-dimensional finite difference time domain (LOD-FDTD) with second-order temporal accuracy (denoted as FLOD2-FDTD). The proposed method can highly improve the performance of the FLOD2-FDTD even when the conductor is embedded into more than half of the cell by the coordinate transformation. The experimental results showed that the proposed method can achieve higher accuracy when the time step size is less than or equal to 5 times of that the Courant-Friedrich-Levy (CFL) condition allowed. In comparison with the previously reported methods, the proposed method for calculating electromagnetic field near microstrip line edge not only improves the efficiency, but also can provide a higher accuracy.

\section{Introduction}

In recent years, as a direct time-domain method for solving Maxwell differential equations, the finite-difference time domain (FDTD) has been extensively studied and widely used in all types of electromagnetic simulation problems. Since the microstrip line usually has a narrow width and effect of the field singularities near its edge [1], the conventional FDTD requires fine grids to accurately characterize the electromagnetic field near microstrip line edge. Furthermore, due to the CFL condition, fine grids would need small time step size, which results in a large resource usage for the conventional FDTD in calculating the microstrip structure.

For these reasons, Taflove proposed a subgrid technique [2] which used fine grids near the edge of microstrip line to characterize a sharp variation of the electromagnetic field. Furthermore, coarse grids were employed for the gently varying electromagnetic field to reduce the number of the FDTD unit cells. However, the time step size was limited by the finest grid size according to the CFL condition, and hence the improvement in computational efficiency was not obvious. Railton proposed the modified assigned material parameters (MAMPs) method [3], which was implemented by the use of an appropriate permittivity and permeability on the material to characterize the singular behavior of the field near the edge. Although the MAMPs can provide a higher accuracy, without reducing the grid size, it was difficult to deal with the situation when the conductor was embedded into more than half of the cell and its computational efficiency was still limited by the CFL condition. Thus, the CFL condition degrades the performance of the conventional FDTD.

To overcome the CFL constraint, several unconditionally stable FDTD methods such as Crank Nicolson FDTD (CNFDTD) [4-7], alternating direction implicit FDTD (ADIFDTD) [8-11], and, more recently, locally one-dimensional FDTD (LOD-FDTD) [12-20] have been proposed. In particular, the LOD-FDTD has simpler implementation and higher efficiency than the other unconditionally stable FDTD. However, the conventional LOD-FDTD exhibits an extra noncommutativity error term, which makes it accurate to first order only in time. In order to achieve second-order temporal accuracy, an improved LOD-FDTD named fundamental LOD-FDTD with second-order temporal accuracy (FLOD2FDTD) has been reported in [16].

In this paper, a new method is proposed by incorporating the quasistatic analytical expressions of electric and magnetic 


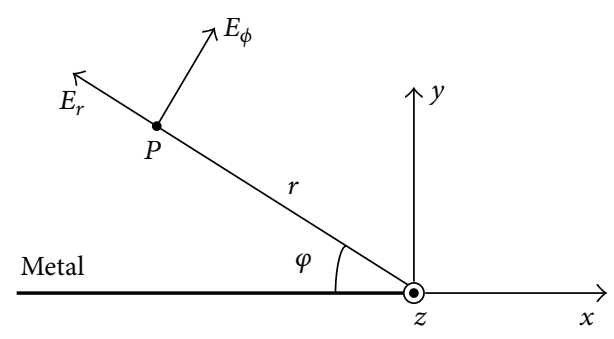

FIGURE 1: Transverse electric and magnetic field components around a microstrip line edge.

fields near the microstrip line edge given by van Bladel [21] into the FLOD2-FDTD. Also, the proposed method can deal with the situation when the conductor is embedded into more than half of the cell by the coordinate transformation and hence has a wider applicability for solving the electromagnetic problems. The experimental results demonstrated that the proposed method not only maintains high computational efficiency by using the FLOD2-FDTD, but also has high accuracy by introducing a priori knowledge of the field near microstrip line edge.

The present paper is organized as follows. Section 2 introduces the proposed method based on the quasistatic analytical expressions and the FLOD2-FDTD and then presents the expressions of the electromagnetic fields near microstrip line edge. Section 3 provides a series of experimental results by the use of proposed method in comparison with the subgrid technique, MAMPs, and FLOD2-FDTD. In addition, the performance of proposed method with different grid sizes and time step sizes is also given and discussed in detail. Section 4 gives a brief summarization of the paper.

\section{Proposed Singularity Processing Method}

The quasistatic electromagnetic fields near a metal edge are given by van Bladel. For the structure shown in Figure 1, the transverse field components in a plane perpendicular to the edge are expressed as [21]

$$
\begin{aligned}
& E_{r}=-\frac{B}{2 \sqrt{r}} \sin \left(\frac{\varphi}{2}\right), \\
& H_{r}=-\frac{A}{2 \sqrt{r}} \cos \left(\frac{\varphi}{2}\right), \\
& E_{\varphi}=-\frac{B}{2 \sqrt{r}} \cos \left(\frac{\varphi}{2}\right), \\
& H_{\varphi}=\frac{A}{2 \sqrt{r}} \sin \left(\frac{\varphi}{2}\right),
\end{aligned}
$$

where $r$ is the distance between the edge and the field point, $\varphi$ is the angle measured from the metal sheet, and $A, B$ are unknown constants. The transverse field components tend to infinity when $r=0$; that is, they are singular.

Here, a general structure of microstrip line shown in Figure 2 is considered to discuss the proposed method. In these figures, Cartesian coordinate is used, and the parameters $\Delta x, \Delta y$, and $\Delta z$ are the spatial step sizes in $x, y$, and $z$ axes, respectively, while the $i, j$, and $k$ are the serial numbers of the cell. From Figure 2(a), we can see that a metal with a length of $s$ is embedded into the cell and $s>\Delta x / 2$. Note that $E_{x}$ and $H_{y}$ tend to zero according to the conventional FDTD, which is equivalent to increasing the length of the metal embedded into the cell, and that will cause calculation errors. The electric and magnetic fields are defined as $E_{x}(i, j, k), E_{x}(i, j, k+1)$, and $H_{y}(i, j, k)$, respectively, for a new location, which is away from the original location by a distance of $s / 2$ in the $x$-axis. Then, we have $r=(\Delta x-s) / 2, \varphi=180^{\circ}$, and $H_{y}(i, j, k)=-H_{\varphi}$, $E_{x}(i, j, k)=E_{r}$. From (1a) and (1d), we get

$$
\begin{aligned}
& B=-\sqrt{2(\Delta x-s)} E_{x}(i, j, k), \\
& A=-\sqrt{2(\Delta x-s)} H_{y}(i, j, k) .
\end{aligned}
$$

Substituting (2a) and (2b) into (1a) and (1d), respectively, the distributions of the transverse field near the metal edge are expressed as

$$
\begin{gathered}
E_{x}(r, j, k)=\sqrt{\frac{\Delta x-s}{2 r}} E_{x}(i, j, k), \\
H_{y}(r, j, k)=\sqrt{\frac{\Delta x-s}{2 r}} H_{y}(i, j, k) .
\end{gathered}
$$

Similarly, $H_{x}$ and $H_{y}$ shown in Figure 2(b) can be divided into six segments according to the angle $\varphi$, which is represented by

$$
\begin{aligned}
& a b: H_{x}=\sin (\pi-\varphi) \frac{A_{1}}{\sqrt{(2 \Delta y / \sin (\pi-\varphi))}} \sin \frac{\varphi}{2}, \\
& b c: H_{y}=-\cos (\pi-\varphi) \frac{A_{2}}{\sqrt{((6 \Delta x-4 s) / \cos (\pi-\varphi))}} \sin \frac{\varphi}{2},
\end{aligned}
$$

$$
c d: H_{y}=-\cos (\varphi-\pi) \frac{A_{2}}{\sqrt{((6 \Delta x-4 s) / \cos (\varphi-\pi))}} \sin \frac{\varphi}{2},
$$

$$
\begin{aligned}
& \text { de }: H_{x}=-\sin (\varphi-\pi) \frac{A_{3}}{\sqrt{(2 \Delta y / \sin (\varphi-\pi))}} \sin \frac{\varphi}{2}, \quad(4 \mathrm{~d}) \\
& \text { ef }: H_{y}=-\cos (\varphi-\pi) \frac{A_{4}}{\sqrt{(2(\Delta x-s) / \cos (\varphi-\pi))}} \sin \frac{\varphi}{2},
\end{aligned}
$$

$$
f a: H_{y}=-\cos (\pi-\varphi) \frac{A_{4}}{\sqrt{(2(\Delta x-s) / \cos (\pi-\varphi))}} \sin \frac{\varphi}{2},
$$




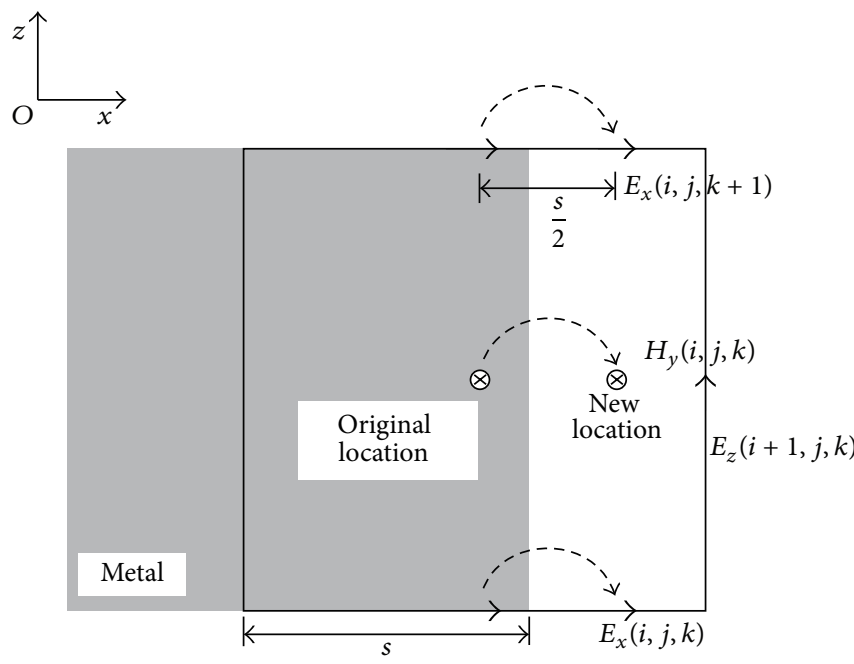

(a)

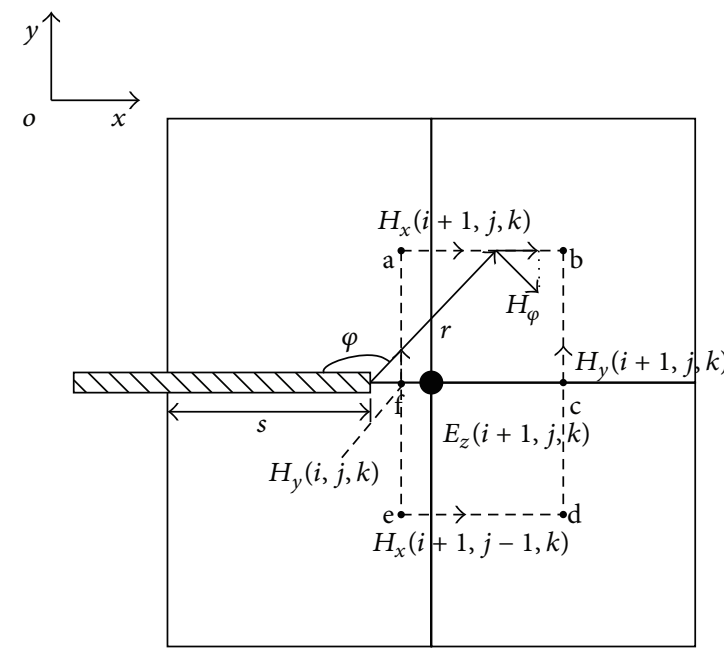

(b)

FIGURE 2: Metal embedded in the cell.

where

$$
A_{1}=\frac{2\left[\left(\Delta y^{2} / 4\right)+(\Delta x-s)^{2}\right]^{5 / 4}}{\sqrt{\Delta y \sqrt{\left(\Delta y^{2} / 4\right)+(\Delta x-s)^{2}}+\Delta x-s}} H_{x}(i+1, j, k)
$$$$
A_{2}=-\sqrt{6 \Delta x-4 s} H_{y}(i+1, j, k) \text {, }
$$

$$
\begin{aligned}
A_{3}= & \frac{2\left[\left(\Delta y^{2} / 4\right)+(\Delta x-s)^{2}\right]^{5 / 4}}{\sqrt{\Delta y \sqrt{\left(\Delta y^{2} / 4\right)+(\Delta x-s)^{2}}+\Delta x-s}} \\
& \times H_{x}(i+1, j-1, k), \\
A_{4}= & -\sqrt{2(\Delta x-s)} H_{y}(i, j, k) .
\end{aligned}
$$

On the basis of the obtained expressions for the electromagnetic field near microstrip line edge, our proposed method can accurately express the fields without reducing the grid size. However, the computational efficiency is still limited by the CFL condition. In order to remove the CFL condition, the unconditionally stable method LOD-FDTD is employed to incorporate with the obtained expressions for the electromagnetic field near microstrip line edge. Although conventional LOD-FDTD can provide high efficiency, it exhibits an extra noncommutativity error term, making it accurate to first order only in time. To achieve secondorder temporal accuracy, we employed the FLOD2-FDTD given in [16] which has second-order temporal accuracy to obtain better accuracy in dealing with the electromagnetic field near microstrip line edge. FLOD2-FDTD is an efficient implementation of LOD-FDTD with second-order temporal accuracy (denoted as LOD2-FDTD) based on the fundamental schemes. The update equations of the LOD2-FDTD are given as follows:

$$
\begin{aligned}
& \left(\mathbf{I}-\frac{\Delta t}{2} \mathbf{A}\right) \mathbf{u}^{n+(3 / 4)}=\left(\mathbf{I}+\frac{\Delta t}{2} \mathbf{A}\right) \mathbf{u}^{n+(1 / 4)}, \\
& \left(\mathbf{I}-\frac{\Delta t}{2} \mathbf{B}\right) \mathbf{u}^{n+(5 / 4)}=\left(\mathbf{I}+\frac{\Delta t}{2} \mathbf{B}\right) \mathbf{u}^{n+(3 / 4)},
\end{aligned}
$$

where

$$
\begin{aligned}
\mathbf{A} & =\left[\begin{array}{cccccc}
0 & 0 & 0 & 0 & 0 & \frac{1}{\varepsilon} \partial_{y} \\
0 & 0 & 0 & \frac{1}{\varepsilon} \partial_{z} & 0 & 0 \\
0 & 0 & 0 & 0 & \frac{1}{\varepsilon} \partial_{x} & 0 \\
0 & \frac{1}{\mu} \partial_{z} & 0 & 0 & 0 & 0 \\
0 & 0 & \frac{1}{\mu} \partial_{x} & 0 & 0 & 0 \\
\frac{1}{\mu} \partial_{y} & 0 & 0 & 0 & 0 & 0
\end{array}\right], \\
\mathbf{B} & =\left[\begin{array}{cccccc}
0 \\
0 & 0 & 0 & 0 & \frac{-1}{\varepsilon} \partial_{z} & 0 \\
0 & 0 & 0 & 0 & 0 & \frac{-1}{\varepsilon} \partial_{x} \\
0 & 0 & \frac{-1}{\mu} \partial_{y} & 0 & 0 & 0 \\
\frac{-1}{\mu} \partial_{z} & 0 & 0 & 0 & 0 & 0 \\
0 & \frac{-1}{\mu} \partial_{x} & 0 & 0 & 0 & 0 \\
E_{x} & E_{y} & E_{z} & H_{x} & H_{y} & H_{z}
\end{array}\right],
\end{aligned}
$$


Based on the LOD2-FDTD principle and the distributions of transverse field near the metal edge, the two procedures for updating the electric field $E_{z}$ and magnetic field $H_{y}$ near microstrip line edge can be formulated as follows (the equations for other field components can be written similarly).

The first procedure is from $n+1 / 4$ to $n+3 / 4$. From (6a), we obtain

$$
E_{z}^{n+(3 / 4)}-\frac{\Delta t}{2 \varepsilon} \frac{\partial H_{y}^{n+(3 / 4)}}{\partial x}=E_{z}^{n+(1 / 4)}+\frac{\Delta t}{2 \varepsilon} \frac{\partial H_{y}^{n+(1 / 4)}}{\partial x} .
$$

Since $H_{y}(i, j, k)$ and $H_{y}(i+1, j, k)$ vary with the angle $\varphi$, we turn (8) into an integral form and then get

$$
\begin{aligned}
& \iint_{S_{1}} E_{z}^{n+(3 / 4)}(i+1, j, k) \mathrm{d} s \\
& -\frac{\Delta t}{2 \varepsilon}\left[\int_{b c+c d} H_{y}^{n+(3 / 4)}(i+1, j, k) \mathrm{d} \varphi\right.
\end{aligned}
$$

$$
\begin{aligned}
E_{z}^{n+(3 / 4)}(i+1, j, k)-\frac{\Delta t}{2 \varepsilon(\Delta x-(s / 2)) \Delta y} & \\
\times & {\left[\int_{\pi-\arctan (\Delta y /(3 \Delta x-2 s))}^{\pi+\arctan (\Delta y /(3 \Delta x-2 s))}-\cos (\varphi-\pi) \frac{A_{2}^{n+(3 / 4)}}{\sqrt{((6 \Delta x-4 s) / \cos (\varphi-\pi))}} \sin \frac{\varphi}{2} \mathrm{~d} \varphi\right.} \\
= & \left.\quad E_{z}^{n+(1 / 4)}(i+1, j, k)+\frac{A_{4}^{n+(3 / 4)}}{2 \varepsilon(\Delta x-\arctan (\Delta y /(\Delta x-s))} \cos (\varphi-\pi) \frac{\Delta t}{\sqrt{(2(\Delta x-s) / \cos (\varphi-\pi))}} \sin \frac{\varphi}{2} \mathrm{~d} \varphi\right] \\
\times & {\left[\int_{\pi-\arctan (\Delta y /(3 \Delta x-2 s))}^{\pi+\arctan (\Delta y /(3 \Delta x-2 s))}-\cos (\varphi-\pi) \frac{A_{2}^{n+(1 / 4)}}{\sqrt{((6 \Delta x-4 s) / \cos (\varphi-\pi))}} \sin \frac{\varphi}{2} \mathrm{~d} \varphi\right.} \\
& \left.+\int_{\pi-\arctan (\Delta y /(\Delta x-s))}^{\pi+\arctan (\Delta y /(\Delta x-s))} \cos (\varphi-\pi) \frac{A_{4}^{n+(1 / 4)}}{\sqrt{(2(\Delta x-s) / \cos (\varphi-\pi))}} \sin \frac{\varphi}{2} \mathrm{~d} \varphi\right],
\end{aligned}
$$

where $S_{1}$ is the area of the rectangular contour around $E_{z}(i+$ $1, j, k)$. Substituting (4a), (4b), (4c), (4d), (4e), and (4f) into (9), we obtain where $\Delta t$ is time step size and $\varepsilon$ is dielectric constant.

The update equations for the field component $H_{y}(i, j, k)$ nearest the edge are derived from the Faradays Law and are given by

$$
\begin{aligned}
& H_{y}^{n+(3 / 4)}(i, j, k)-\frac{\Delta t}{2 \sqrt{2} \mu(\Delta x-s)} E_{z}^{n+(3 / 4)}(i+1, j, k) \\
& \quad=H_{y}^{n+(1 / 4)}(i, j, k)+\frac{\Delta t}{2 \sqrt{2} \mu(\Delta x-s)} E_{z}^{n+(1 / 4)}(i+1, j, k),
\end{aligned}
$$

$$
\begin{aligned}
H_{y}^{n+(3 / 4)}(i+1, j, k) & \\
& \quad-\frac{\Delta t}{2 \mu \Delta x}\left[E_{z}^{n+(3 / 4)}(i+2, j, k)-E_{z}^{n+(3 / 4)}(i+1, j, k)\right] \\
= & H_{y}^{n+(1 / 4)}(i+1, j, k) \\
& +\frac{\Delta t}{2 \mu \Delta x}\left[E_{z}^{n+(1 / 4)}(i+2, j, k)-E_{z}^{n+(1 / 4)}(i+1, j, k)\right],
\end{aligned}
$$

where $\mu$ is permeability. Substituting (5a), (5b), (5c), (5d), (11), 
modify the scheme of the LOD2-FDTD into the fundamental scheme, then we get

$$
\begin{aligned}
{\left[\frac{1}{2}+\right.} & \frac{m \Delta t^{2}}{8 \mu \varepsilon(\Delta x-(s / 2)) \Delta x \Delta y} \\
& \left.+\frac{n \Delta t^{2}}{8 \sqrt{2} \mu \varepsilon(\Delta x-(s / 2))(\Delta x-s) \Delta y}\right] \\
& \times e_{z}^{n+(3 / 4)}(i+1, j, k) \\
& -\frac{m \Delta t^{2}}{8 \mu \varepsilon(\Delta x-(s / 2)) \Delta x \Delta y} e_{z}^{n+(3 / 4)}(i+2, j, k) \\
= & E_{z}^{n+(1 / 4)}(i, j, k) \\
& +\frac{\Delta t}{2 \varepsilon(\Delta x-(s / 2)) \Delta y} \\
& \times\left[m H_{y}^{n+(1 / 4)}(i+1, j, k)-n H_{y}^{n+(1 / 4)}(i, j, k)\right],
\end{aligned}
$$

where

$$
\begin{gathered}
e_{z}^{n+(3 / 4)}=E_{z}^{n+(3 / 4)}+E_{z}^{n+(1 / 4)}, \\
m=\int_{-\arctan (\Delta y /(3 \Delta x-2 s))}^{\arctan (\Delta y /(3 \Delta x-2 s))} \cos ^{3 / 2} \varphi \cos \frac{\varphi}{2} \mathrm{~d} \varphi, \\
n=\int_{-\arctan (\Delta y /(\Delta x-s))}^{\arctan (\Delta y /(\Delta x-s))} \cos ^{3 / 2} \varphi \cos \frac{\varphi}{2} \mathrm{~d} \varphi .
\end{gathered}
$$

From the discussions above, we can get $E_{z}^{n+(3 / 4)}$ by the use of (13) and (14). Then, $H_{y}^{n+(3 / 4)}$ can be obtained from (11). The second procedure is from $n+3 / 4$ to $n+5 / 4$. Similarly, we have

$$
\begin{aligned}
- & \frac{p \Delta t^{2}}{8 \mu \varepsilon(\Delta x-(s / 2)) \Delta y^{2}} e_{z}^{n+(5 / 4)}(i+1, j-1, k) \\
& +\left[\frac{1}{2}+\frac{p \Delta t^{2}}{4 \mu \varepsilon(\Delta x-(s / 2)) \Delta y^{2}}\right] e_{z}^{n+5 / 4}(i+1, j, k) \\
& -\frac{p \Delta t^{2}}{8 \mu \varepsilon(\Delta x-(s / 2)) \Delta y^{2}} e_{z}^{n+(5 / 4)}(i+1, j+1, k) \\
= & E_{z}^{n+(3 / 4)}(i+1, j, k) \\
& -\frac{p \Delta t}{2 \varepsilon(\Delta x-(s / 2)) \Delta y} \\
& \times\left[H_{x}^{n+(3 / 4)}(i+1, j, k)-H_{x}^{n+(3 / 4)}(i+1, j-1, k)\right],
\end{aligned}
$$

where

$$
\begin{aligned}
p= & \int_{\arctan (\Delta y /(3 \Delta x-2 s))}^{\arctan (\Delta y /(\Delta x-s))} \sin ^{3 / 2} \varphi \cos \frac{\varphi}{2} \mathrm{~d} \varphi \\
& \times \frac{2\left[\left(\Delta y^{2} / 4\right)+(\Delta x-s)^{2}\right]^{5 / 4}}{\sqrt{\Delta y\left[\sqrt{\left(\Delta y^{2} / 4\right)+(\Delta x-s)^{2}}+\Delta x-s\right]}} .
\end{aligned}
$$

Thus, the update equation of $H_{y}(i, j, k)$ is given by

$$
\begin{aligned}
& H_{y}^{n+(5 / 4)}(i, j, k) \\
& =H_{y}^{n+(3 / 4)}(i, j, k) \\
& -\frac{\Delta t}{2 \mu \Delta z}\left[E_{x}^{n+(5 / 4)}(i, j, k+1)+E_{x}^{n+(3 / 4)}(i, j, k+1)\right. \\
& \left.\quad-E_{x}^{n+(5 / 4)}(i, j, k)-E_{x}^{n+(3 / 4)}(i, j, k)\right] .
\end{aligned}
$$

Since $m, n$, and $p$ are not time varying, they are calculated once at the beginning of the iterative. Thus, the proposed method will not reduce the computational efficiency. The update equations for other fields away from the microstrip line and the input and output processing are the same as the FLOD2-FDTD shown in [16].

\section{Numerical Results and Discussion}

In this section, to demonstrate the performance of our proposed method, a microstrip line and a microstrip antenna are analyzed. A microstrip line above a ground plane is modeled in order to verify the accuracy of the proposed method, comparing with subgrid technique and MAMPs. The microstrip line is placed at a height of $2 \mathrm{~mm}$ above the ground plane, and the relative dielectric constant of substrate is $\varepsilon_{r}=4$. All the conductors are assumed to be perfect electric conductors (PECs). The microstrip line is terminated at the end by a 400 resistor. A raised cosine pulse as excitation is launched along the microstrip line. The proposed method and MAMPs take a square grid of size $0.5 \mathrm{~mm}$ calculating the characteristic impedance of the microstrip line structure. Based on the principle of the subgrid technique, fine grid with the size of $0.1 \mathrm{~mm}$ is adopted in the region of the microstrip line to guarantee accuracy, while coarse grid with the size of $0.5 \mathrm{~mm}$ is adopted where electromagnetic field varies gently to guarantee computational efficiency. Time step sizes are chosen by the CFL condition. The computational domain is terminated with the Mur absorbing boundary condition in [22]. The characteristic impedance is calculated by comparing the amplitude of the incident pulse with the amplitude of the pulse reflected from the resistor.

Figure 3 shows the characteristic impedances obtained by the proposed method, MAMPs, and subgrid technique. The $s$ is the length of conductor embedded in the cell. It can be seen that the subgrid technique is unable to resolve subcellular changes in length of conductor embedded in, except when the microstrip line edge intersects the point of electric or magnetic field by coincidence, and the characteristic impedance obtained varies widely from the quasistatic result. The average error of subgrid technique compared with the quasistatic result is $3.43 \%$. When $s$ is less than $0.5 \Delta x$, MAMPs follow the quasistatic result closely, and the average error compared with the quasistatic result is $1.91 \%$. But when $s$ is larger than $0.5 \Delta x$, the characteristic impedance obtained by MAMPs does not vary with variation of $s$. In contrast, the proposed method follows the quasistatic result closely, even when $s$ is larger 


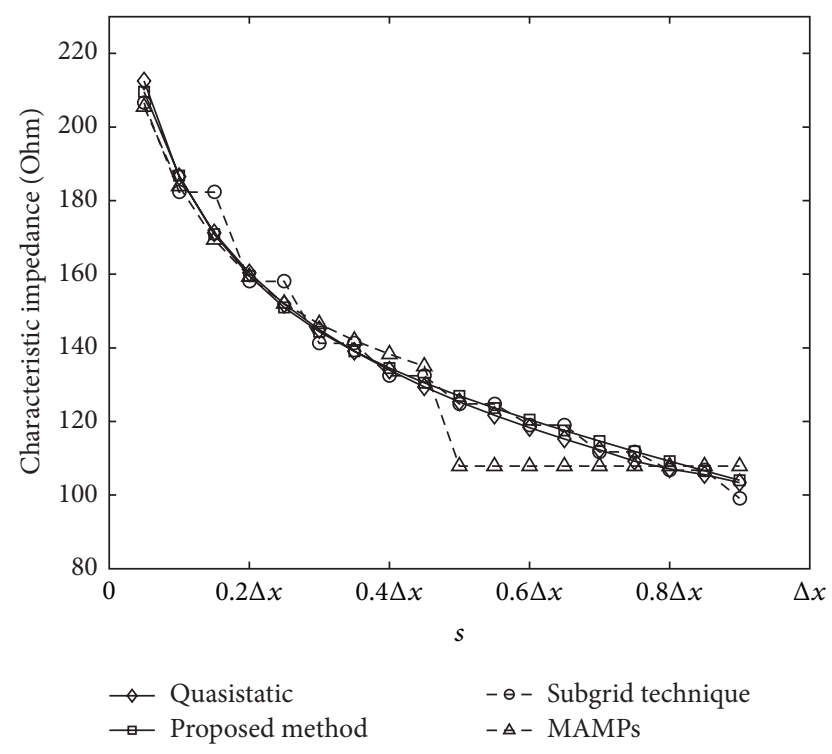

FIGURE 3: Calculated results of different methods.

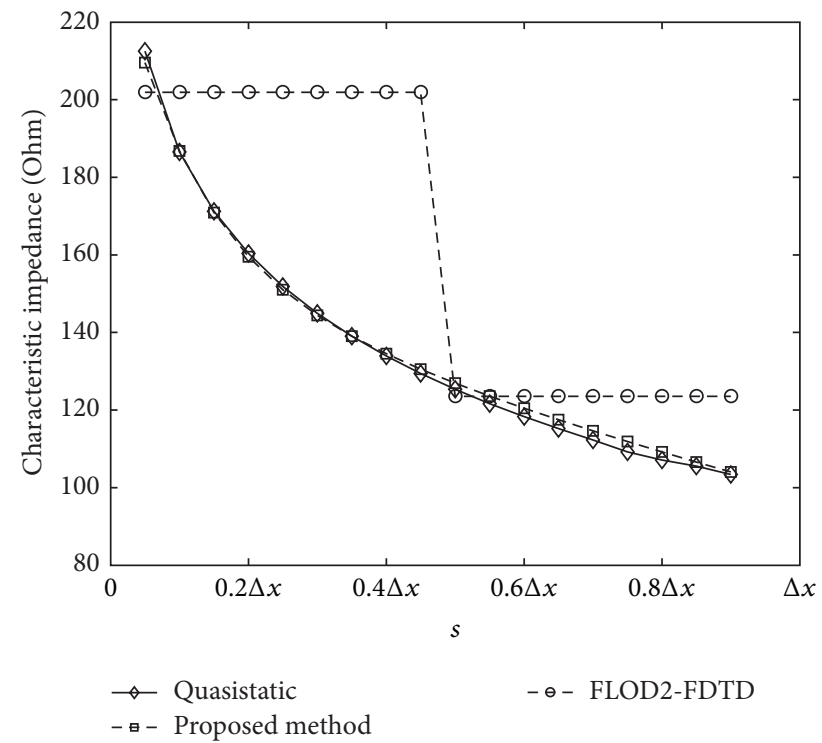

FIGURE 4: Calculated results of the proposed method and the FLOD2-FDTD with the same grid size and CFLN.

than $0.5 \Delta x$, indicating that it is capable of highly accurate characterizations of microstrip structures and can deal with the situation when conductor is embedded into more than half of the cell. The average error of the proposed method compared with the quasistatic formula is $1.08 \%$.

To demonstrate the improvement on the accuracy of the proposed method by introducing the quasistatic analytical expressions into the FLOD2-FDTD, the proposed method with a square grid of size $0.5 \mathrm{~mm}$ and CFLN $=1$ and the FLOD2-FDTD with the same grid size and CFLN are used to analyze the same structure as in Figure 3. The CFL number (CFLN) is the ratio between the time step taken and CFL time limit.

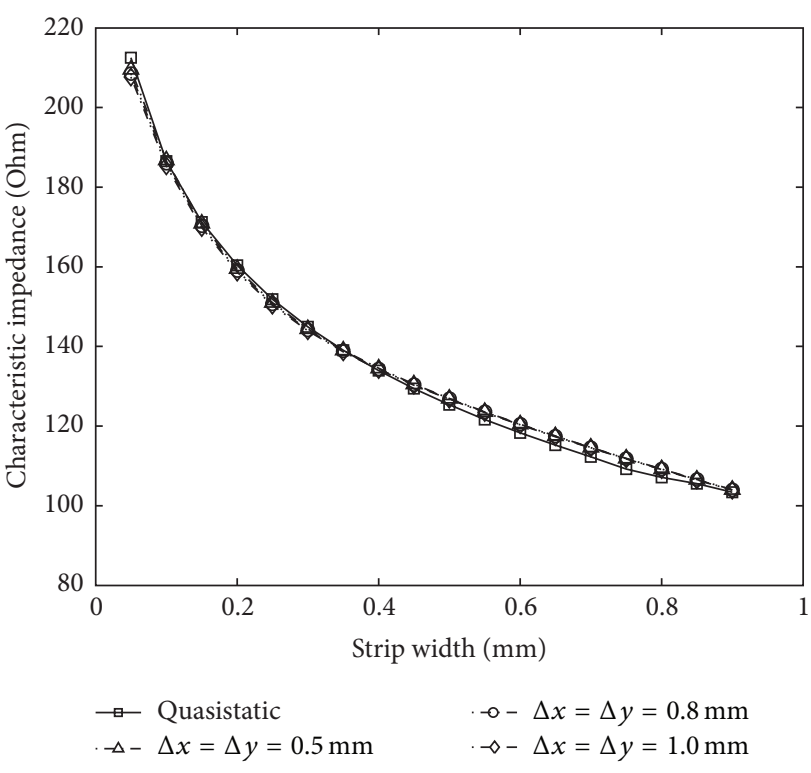

FIGURE 5: Calculated results of the proposed method with different grid sizes.

Figure 4 demonstrates that, like the subgrid technique, the FLOD2-FDTD is also unable to resolve subcellular changes in length of conductor embedded in, except when the microstrip line edge intersects the point of electric or magnetic field coincidently, and the characteristic impedance obtained varies more widely from the quasistatic result. In contrast, the introduction of the quasistatic analytical expressions for electric and magnetic fields makes the proposed method follow the quasistatic result closely and hence has a higher accuracy than the FLOD2-FDTD.

In order to investigate the effect of the grid size and CFLN on the performance of the proposed method, the characteristic impedances of the proposed method with varying grid sizes and CFLNs are shown in Figures 5 and 6 , respectively. Figure 5 shows the effects of the grid size when it is set as $\Delta y=0.5 \mathrm{~mm}, \Delta x=\Delta z=0.5,0.8$, and $1.0 \mathrm{~mm}$, respectively, and CFLN $=1$. The average errors of characteristic impedances obtained from the proposed method with different cell sizes are $1.08 \%, 1.13 \%$, and $1.17 \%$, which are negligible increments. Figure 6 illustrates the characteristic impedances of the proposed method with a square grid size of $0.5 \mathrm{~mm}$ and CFLN $=1,5$, and 10 , respectively. It can be seen that the average errors of the characteristic impedances obtained by the proposed method with CFLN $=1,5$, and 10 are $1.08 \%, 1.51 \%$, and $3.80 \%$, respectively. By comparing the results in Figures 3 and 6, we can see that the proposed method has a higher accuracy when CFLN $\leq 5$, while its performance suffers a large effect due to the numerical dispersion when CLFN $>5$, resulting in that the accuracy of the proposed method is slightly lower than the subgrid technique.

In order to assess the effectiveness of the proposed method on a realistic problem, a microstrip antenna shown in Figure 7, which is designed to operate at around $8 \mathrm{GHz}$, is used as a test case. The proposed method, MAMPs, and 


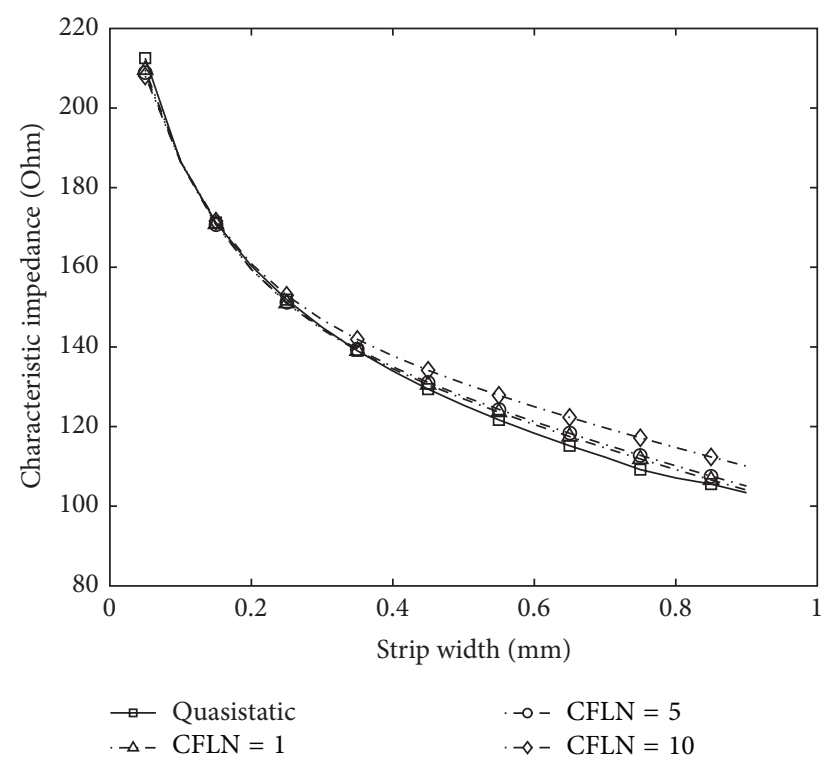

FIGURE 6: Calculated results of the proposed method with different CFLNs.

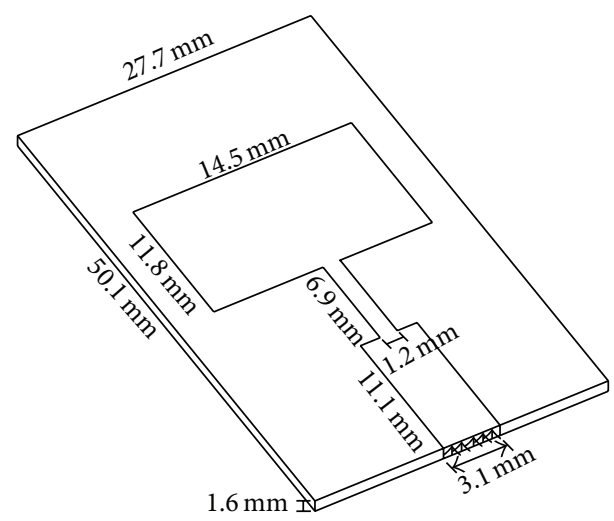

FIGURE 7: Geometry of the microstrip antenna.

conventional FDTD with coarse grid take a square grid of size $0.4 \mathrm{~mm}$, conventional FDTD with fine grid takes a square grid of size $0.1 \mathrm{~mm}$, and subgrid technique takes two square grids of sizes $0.1 \mathrm{~mm}$ and $0.4 \mathrm{~mm}$. Time step sizes are chosen as the value determined by the CFL condition, except the proposed method with CFLN $=5$ which takes 5 times as that of the proposed method with $C F L N=1$. The number of iterations of subgrid technique and conventional FDTD with fine grid is taken to be 20000 , that of the proposed method with CFLN = 1, MAMPs, and conventional FDTD with coarse grid is taken to be 5000, and that of the proposed method with CFLN $=5$ is taken to be 1000 . The computational domain is terminated with the Mur absorbing boundary condition in [22].

The return losses are given in Figure 8. It can be seen that the resonant frequencies obtained by the proposed method with CFLN $=1$ and CFLN $=5$ converge to $8 \mathrm{GHz}$ and agree well with the result obtained by the conventional FDTD with fine grid. Furthermore, the resonant frequencies obtained by MAMPs, subgrid technique, and conventional FDTD

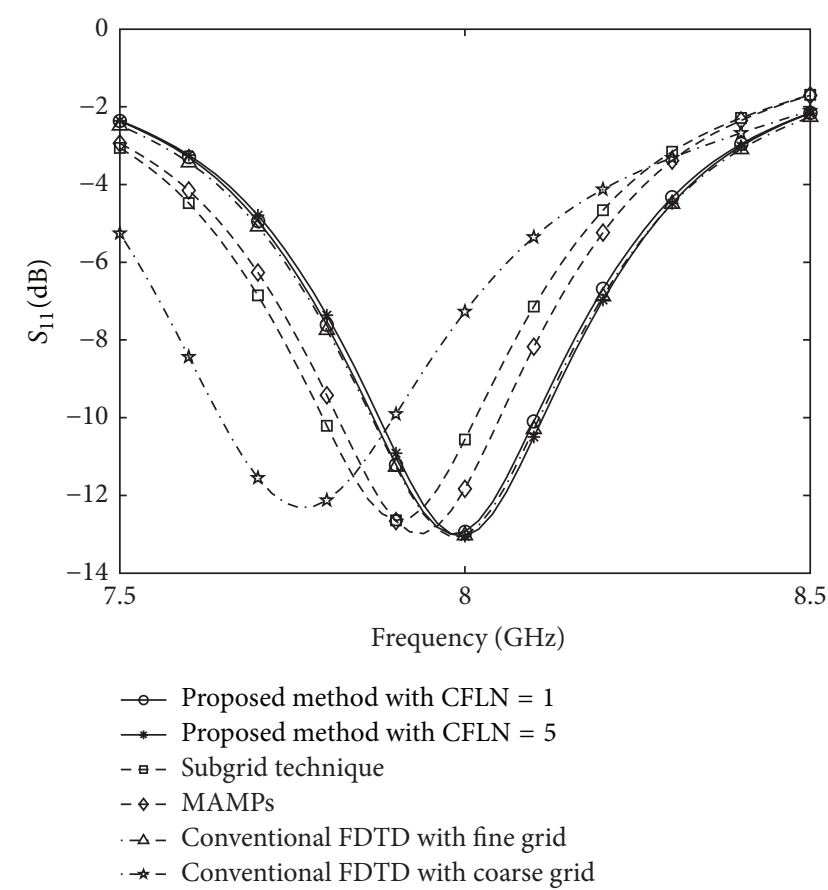

FIGURE 8: Return loss of the microstrip antenna using different methods.

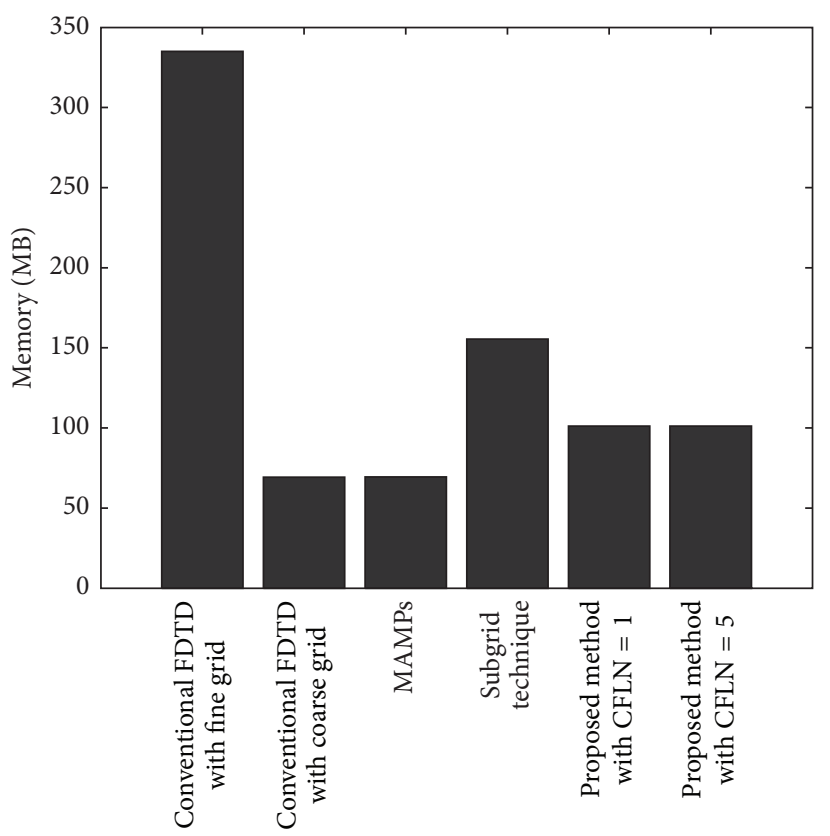

FIGURE 9: Memory requirement using different methods.

with coarse mesh are $7.93 \mathrm{GHz}, 7.91 \mathrm{GHz}$, and $7.78 \mathrm{GHz}$, respectively. The memory requirements for each method are illustrated in Figure 9. In general, the proposed method requires larger memory than the MAMPs and conventional FDTD with coarse mesh owing to the solution of a tridiagonal system of linear equations. However, the proposed method requires smaller memory than subgrid technique and conventional FDTD with fine mesh. The CPU time is presented 


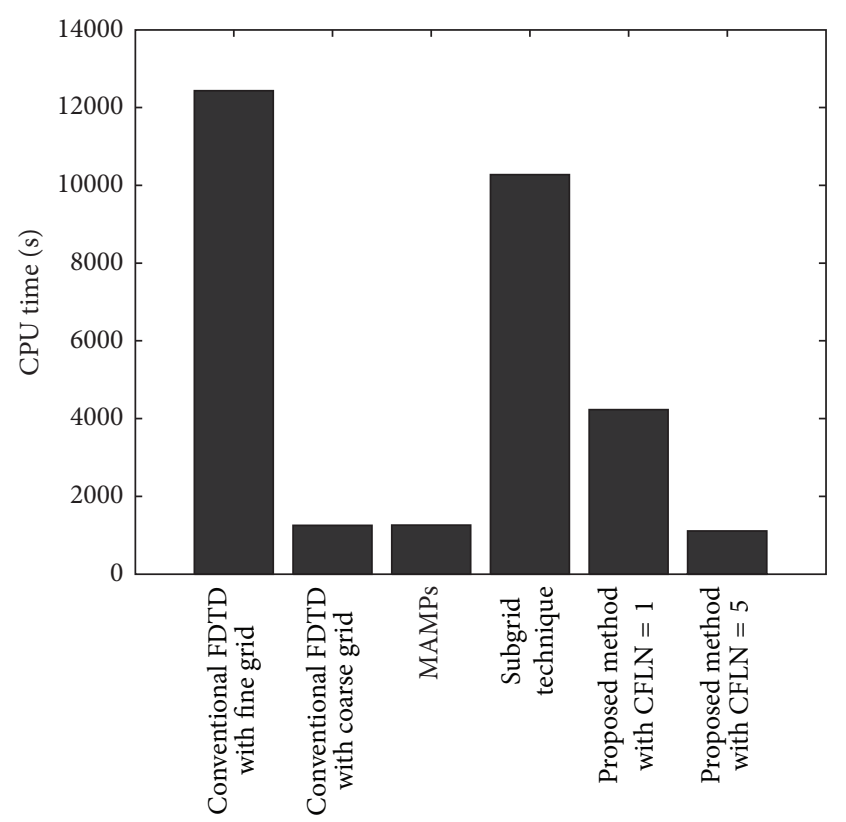

Figure 10: CPU time using different methods.

in Figure 10, where a PC with $3.1 \mathrm{GHz}$ Intel 15 processor and $4 \mathrm{~GB}$ memory is used. It is observed that the time of the proposed method with CFLN $=5$ is shorter than that of the other methods, indicating the high efficiency of the proposed method.

\section{Conclusion}

In this paper, a novel method has been proposed by incorporating the quasistatic analytical expressions into the FLOD2FDTD to analyze the electric and magnetic fields near microstrip line edge and its performance has been studied in detail. The proposed method can deal with the situation when the conductor is embedded into more than half of the cell by the coordinate transformation, making it promising for solving more electromagnetic problems. The experimental results demonstrated that the proposed method with CFLN $\leq 5$ can achieve significantly better accuracy than previously reported methods such as MAMPs, subgrid technique, conventional FDTD, and FLOD2-FDTD. Moreover, our proposed method still has high efficiency without any noticeable computing overhead in memory or time. However, our proposed method is limited by the numerical dispersion. Therefore, ongoing research will hopefully relax this limitation in the future.

\section{Conflict of Interests}

The authors declare that there is no conflict of interests regarding the publication of this paper.

\section{Acknowledgments}

This work was partially supported by the Natural Science Foundation of China (no. 51209055) and the Fundamental Research Funds for the Central Universities (HEUCFD1433).

\section{References}

[1] G. Mur, "The modeling of singularities in the finite-difference approximation of the time-domain electromagnetic-field equations," IEEE Transactions on Microwave Theory and Techniques, vol. 29, no. 10, pp. 1073-1077, 1981.

[2] A. Taflove and S. C. Hagness, Computational Electrodynamics: The Finite-Difference Time-Domain Method, Artech House, Norwood, Mass, USA, 3rd edition, 2005.

[3] C. J. Railton and D. L. Paul, "Analysis of structures containing sharp oblique metal edges in FDTD using MAMPs," IEEE Transactions on Antennas and Propagation, vol. 58, no. 9, pp. 2954-2960, 2010.

[4] W. Wang, D.-M. Zhou, P.-G. Liu, and Y.-J. Qin, “Transient analysis of transmission line with arbitrary loads based on the splitstep Crank-Nicolson-FDTD method," Journal of Electronics and Information Technology, vol. 35, no. 1, pp. 209-214, 2013.

[5] H. Kim, I.-S. Koh, and J.-G. Yook, "Implicit ID-FDTD algorithm based on Crank-Nicolson scheme: dispersion relation and stability analysis," IEEE Transactions on Antennas and Propagation, vol. 59, no. 6, part 2, pp. 2259-2267, 2011.

[6] H. K. Rouf, F. Costen, and M. Fujii, "The modeling of singularities in the finite-difference approximation of the time-domain electromagnetic-field equations," Journal of Electromagnetic Waves and Applications, vol. 25, no. 17-18, pp. 2429-2441, 2011.

[7] T. B. Jin and J. Zou, "Calculation of transmission parameters for a rectangle defected ground structure using fast FDTD scheme," Chinese Journal of Radio Science, vol. 27, no. 5, pp. 853-858, 2012.

[8] S. Yang, Z. Chen, Y. Yu, and S. Ponomarenko, "On the divergence properties of the new efficiency-improved divergence preserved ADI-FDTD method," in Proceedings of the IEEE MTT-S International Microwave Symposium Digest (MTT '13), pp. 1-3, Seattle, Wash, USA, June 2013.

[9] Y.-D. Kong, Q.-X. Chu, and R.-L. Li, "High-order unconditionally-stable two-step leapfrog ADIFDTD methods and numerical analysis," IEEE Transactions on Antennas and Propagation, vol. 61, no. 10, pp. 5135-5143, 2013.

[10] T. H. Gan and E. L. Tan, "Stability and dispersion analysis for three-dimensional (3-D) leapfrog ADI-FDTD method," Progress In Electromagnetics Research M, vol. 23, pp. 1-12, 2012.

[11] S.-C. Yang, Z. Chen, Y. Yu, and W.-Y. Yin, "An unconditionally stable one-step arbitrary-order leapfrog ADI-FDTD method and its numerical properties," IEEE Transactions on Antennas and Propagation, vol. 60, no. 4, pp. 1995-2003, 2012.

[12] G. S. Liu and G. J. Zhang, "Study for the numerical properties of the higher-order LOD-FDTD methods," Journal of Electronics and Information Technology, vol. 32, no. 6, pp. 1384-1388, 2010.

[13] I. Ahmed, E. H. Khoo, and E. Li, "Efficient modeling and simulation of graphene devices with the LOD-FDTD method," IEEE Microwave and Wireless Components Letters, vol. 23, no. 6, pp. 306-308, 2013.

[14] A. K. Saxena and K. V. Srivastava, "Stability and dispersion analysis of higher order unconditionally stable three-step locally onedimensional finite-difference time-domain method," IET Microwaves, Antennas and Propagation, vol. 7, no. 12, pp. 954960, 2013.

[15] F. Zheng and Z. Chen, "Toward the development of a threedimensional unconditionally stable finite-difference timedomain method," IEEE Transactions on Microwave Theory and Techniques, vol. 48, no. 9, pp. 1550-1558, 2000.

[16] E. L. Tan, "Fundamental schemes for efficient unconditionally stable implicit finite-difference time-domain methods," IEEE 
Transactions on Antennas and Propagation, vol. 56, no. 1, pp. 170-177, 2008.

[17] J. Shibayama, M. Muraki, J. Yamauchi, and H. Nakano, "Efficient implicit FDTD algorithm based on locally one-dimensional scheme," Electronics Letters, vol. 41, no. 19, pp. 1046-1047, 2005.

[18] E. L. Tan, "Unconditionally stable LOD-FDTD method for 3-D maxwell's equations," IEEE Microwave and Wireless Components Letters, vol. 17, no. 2, pp. 85-87, 2007.

[19] I. Ahmed, E.-K. Chua, E.-P. Li, and Z. Chen, "Development of the three-dimensional unconditionally stable LOD-FDTD method," IEEE Transactions on Antennas and Propagation, vol. 56, no. 11, pp. 3596-3600, 2008.

[20] T. H. Gan and E. L. Tan, "Unconditionally stable fundamental LOD-FDTD method with second-order temporal accuracy and complying divergence," IEEE Transactions on Antennas and Propagation, vol. 61, no. 5, pp. 2630-2638, 2013.

[21] J. van Bladel, Singular Electromagnetic Fields and Sources, Clarendon, Oxford, UK, 1991.

[22] W. C. Tay and E. L. Tan, "Mur absorbing boundary condition for efficient fundamental 3-D LOD-FDTD," IEEE Microwave and Wireless Components Letters, vol. 20, no. 2, pp. 61-63, 2010. 

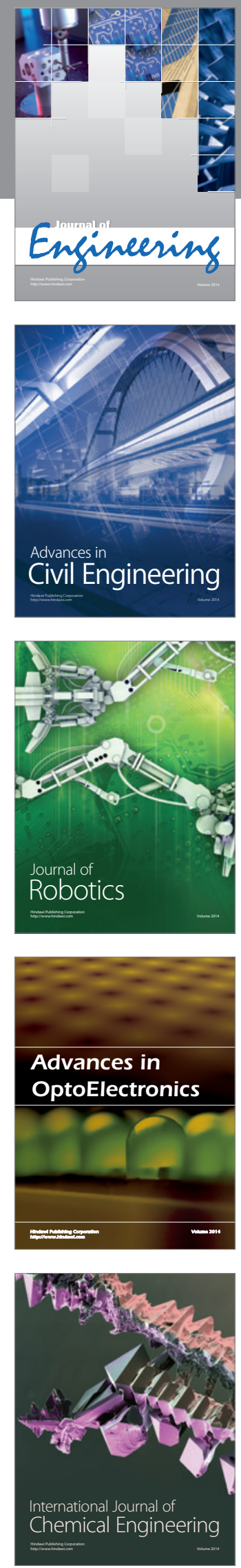

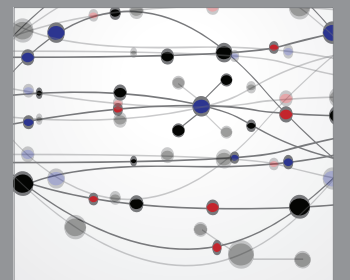

The Scientific World Journal
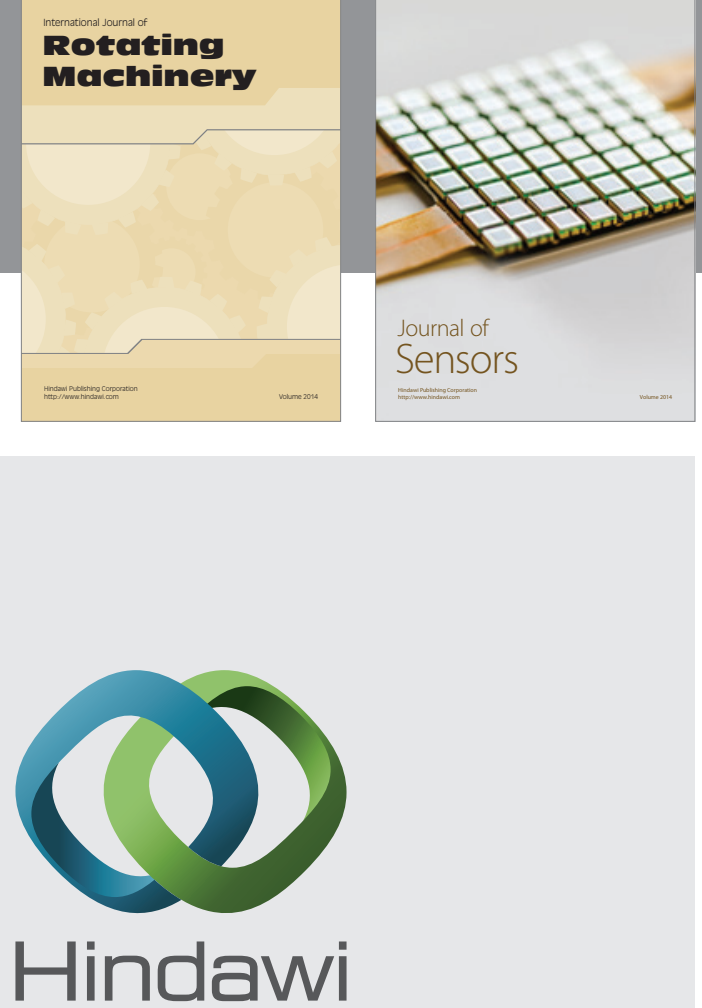

Submit your manuscripts at http://www.hindawi.com
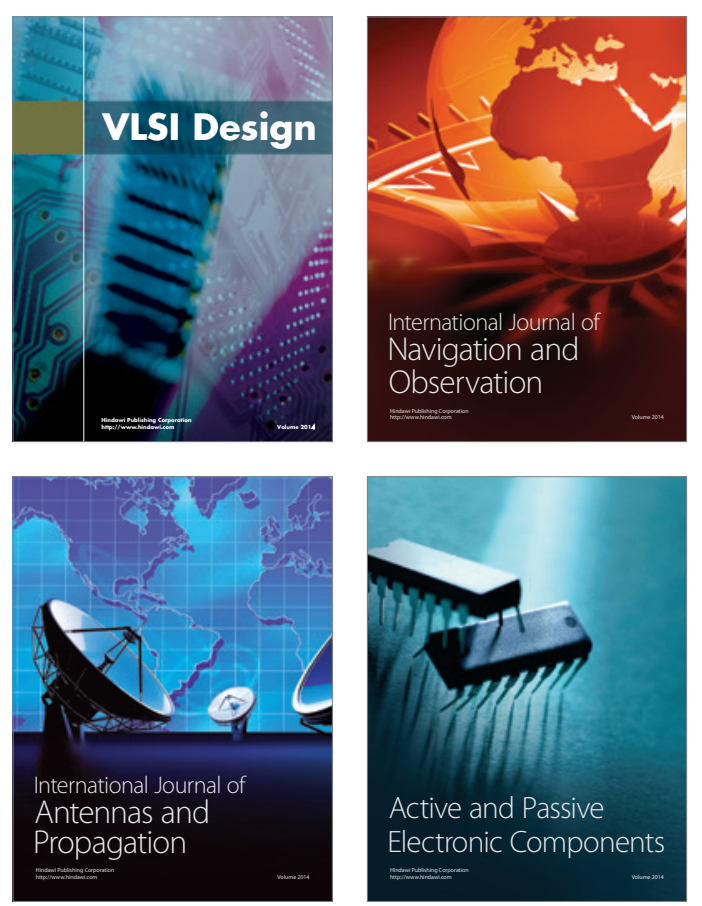
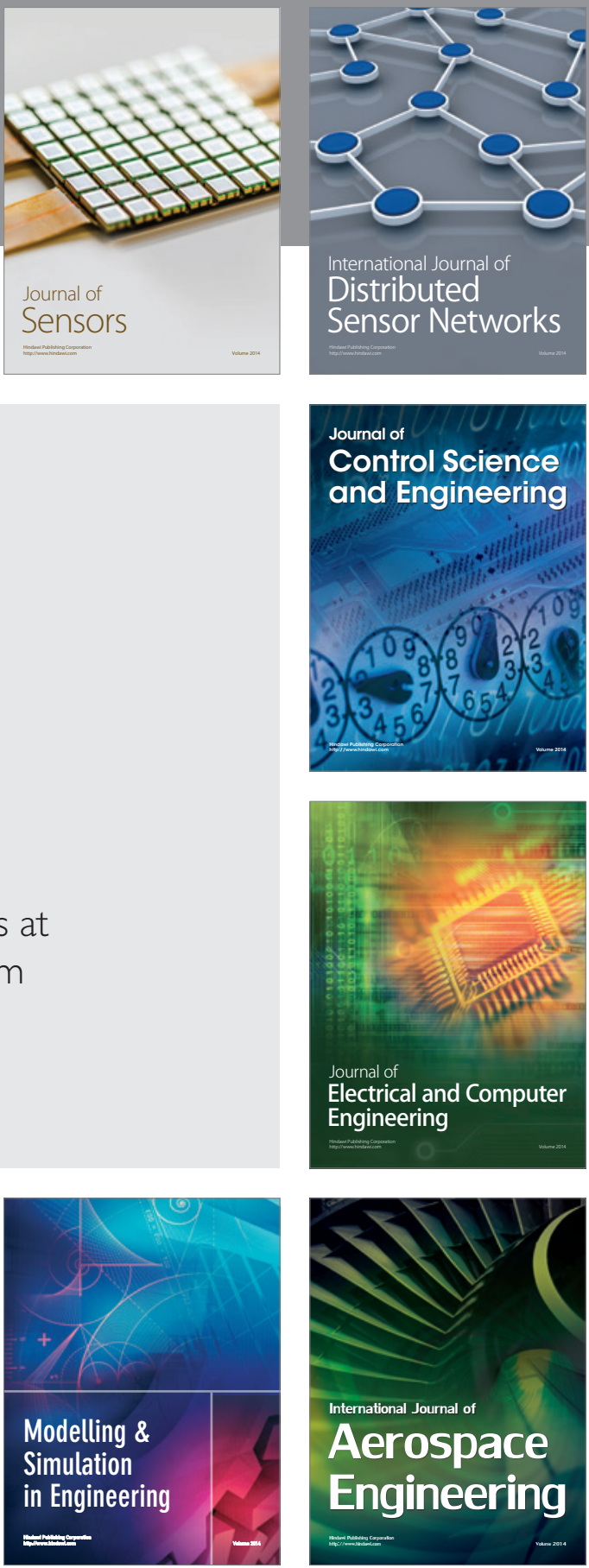

Journal of

Control Science

and Engineering
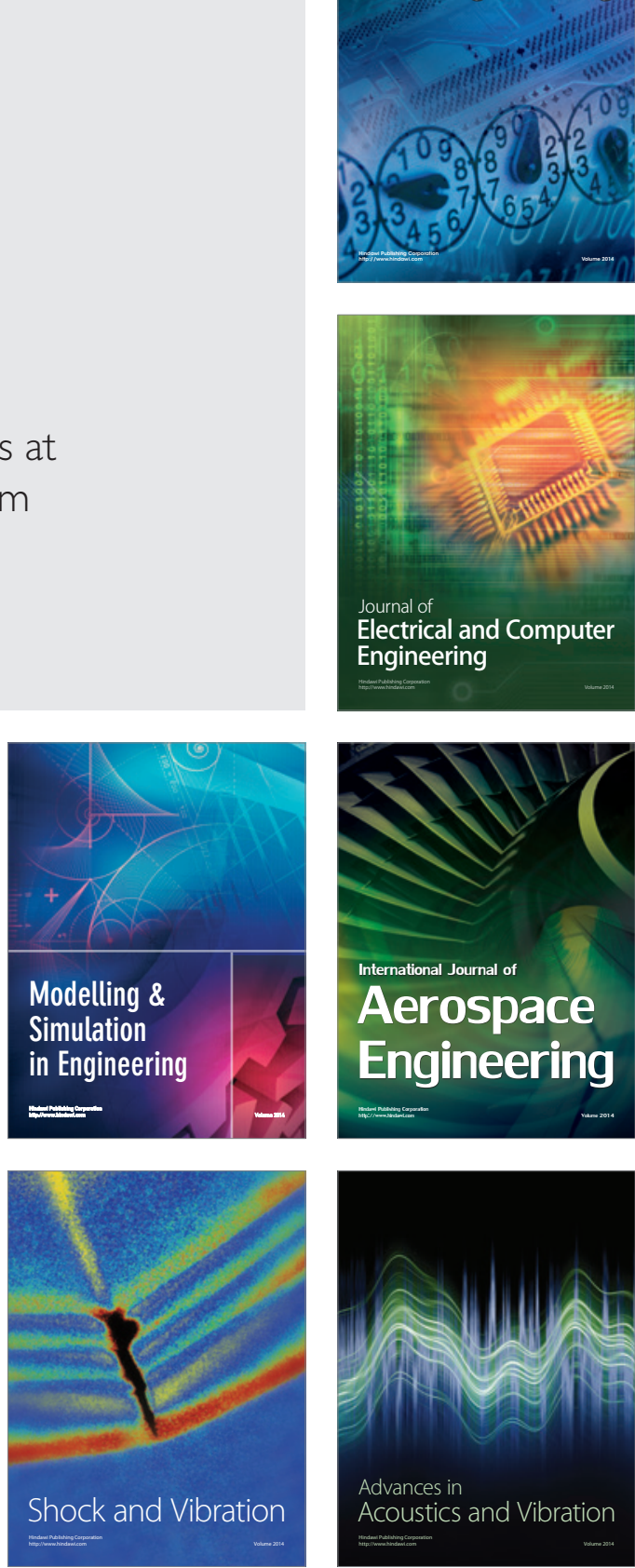